\title{
From herbal small RNAs to one medicine
}

\author{
Chao Ji ${ }^{1}$, Skirmantas Kriaucionis ${ }^{2}$, Benedikt M. Kessler ${ }^{3}$ \& Chengyu Jiang $^{4 *}$ \\ ${ }^{1}$ Institute of Basic Medical Sciences, Chinese Academy of Medical Sciences, Department of Pharmacology, Peking Union Medical College, \\ Beijing 100005, China; \\ ${ }^{2}$ Ludwig Institute for Cancer Research, University of Oxford, OX3 7DQ, UK; \\ ${ }^{3}$ Target Discovery Institute, Nuffield Department of Medicine, CAMS-Oxford Institute, University of Oxford, Roosevelt Drive, Oxford OX3 7FZ, \\ $U K$; \\ ${ }^{4}$ State Key Laboratory of Medical Molecular Biology, Institute of Basic Medical Sciences, CAMS-Oxford Institute, Chinese Academy of Medical \\ Sciences, Department of Biochemistry, Peking Union Medical College, Beijing 100005, China
}

Received February 21, 2019; published online March 1, 2019

Citation: Ji, C., Kriaucionis, S., Kessler, B.M., and Jiang, C. (2019). From herbal small RNAs to one medicine. Sci China Life Sci 62, 285-287. https://doi.org/ 10.1007/s11427-019-9513-y

In this special topic "Herbal small RNAs, novel precision medicine from Mother Nature", we present four original articles that study the entry of small RNAs derived from herbs used in traditional Chinese medicine into mammalian cells and their function within human and mouse cells, and one review paper focusing on current trends in herb genomics (Du et al., 2017; Huang et al., 2018; Zhang et al., 2018; Li et al., 2019; Xin et al., 2018).

Controversy over whether plant-derived microRNAs could enter and function in mammalian cells and organisms began following publications by Zhang Chenyu in 2012 (Zhang et al., 2012) and Dickinson et al. in 2013 (Dickinson et al., 2013). Although the current knowledge may not be able to explain how plant derived small nucleic acids enter mammalian cells and survive the nuclease degradation within, plant-derived small RNAs have been successfully detected in mammalian cells and tissues (Chin et al., 2016; Zhu et al., 2017). Before we believe that regulation by small RNA occurs across phylogenetic kingdoms, three critical questions need to be answered: (1) What is the quantity of plant-derived small RNAs entering mammalian cells and tissues? (2) By which routes does entry occur? (3) What protects small RNAs from degradation after entry?

*Corresponding author (email: jiang@pumc.edu.cn)
In the manuscript "Large-scale analysis of small RNAs derived from traditional Chinese herbs in human tissues", Huang et al. discovered thousands of unique small RNA sequences derived from 10 traditional Chinese medicine herbs in human blood samples and mice lung tissues (Huang et al., 2018). Over $90 \%$ of the small RNAs (sRNAs) sequences from the herbs Chuan Xin Lian and Hong Jing Tian identified in the study could be mapped to herb genomes, confirming their plant origins. These data suggest that large amounts of plant-derived small RNAs can enter mammalian cells and tissues, and the cross-kingdom regulation by microRNAs proposed by Zhang Chenyu group may be a common phenomenon (Zhang et al., 2012).

An online publication by Du et al. in 2017 provided an answer to the question of how plant-derived small RNAs enter mammalian cells and tissues: "Plant-derived phosphocholine facilitates cellular uptake of anti-pulmonary fibrotic HJT-sRNA-m7" (Du et al., 2017) described herbderived phosphocholines able to facilitate the entrance of small RNAs into mammalian cells. Many traditional Chinese medicines are administered by ingestion of a decoction, a concentrated plant essence produced by boiling herbs with water for 30-60 min, and consumed orally by patients. Li et al. discovered that a heat-stable decoctosome - an exosomelike nanoparticle isolated from a decoction and consisting of 
lipids, proteins, chemicals, and small RNAs - could enter mammalian cells and tissues by oral administration. The results are published in this issue: "Herbal decoctosome is a novel form of medicine".

A simple bencaosome, composed of synthetic lipids and sRNAs, is a simulation of a decoctosome constructed for medical applications. Li et al. found that for optimal bencaosome preparation, lipids and small RNAs should be mixed at 90 degrees for $15 \mathrm{~min}$ ( $\mathrm{Li}$ et al., 2019). Using a critical micelle concentration assay, small RNAs in the bencaosome were found to co-assemble with the lipid layers, suggesting that these small RNAs may be protected from degradation. A previous study reported that 2'-O-methyl groups on small interfering RNAs (siRNAs) can stabilize the siRNAs in serum without affecting their activity in mammalian RNA interference (Yu et al., 2005). Plant microRNAs have a natural occurring methyl group on the ribose of the last nucleotide (Khvorova and Watts, 2017), indicating that herbal microRNAs are naturally stable in mammalian cells. Other phosphodiester or ribose modifications may impact both stability and affinity of microRNAs to their targets. The main instability of RNA comes from $2^{\prime} \mathrm{OH}$ group in the ribose. It acts as a nucleophile, reacting with the adjacent phosphate and resulting in cleavage of the RNA backbone. Thus, the ribose or phosphodiester modifications are likely to impact the stability of RNA. Moreover, RNA backbone modifications can impact base-pairing affinity. For example, 2'-O-methylnucleotides have been demonstrated to have elevated melting temperature of resulting duplex molecules (Majlessi et al., 1998). Future studies are necessary to explore additional modifications that might protect sRNAs from nuclease degradation in cells.

In this special topic, the molecular mechanisms of some herbal-derived small RNAs activities were elucidated. PGYsRNA-6 was identified in a screen of the anti-inflammatory herb Pu Gong Ying and shown to target the RELA gene, a component of transcription factor NF- $\mathrm{kB}$ (Li et al., 2019). Bencaosomes with PGY-sRNA-6 inhibited poly(I:C) induced inflammation both in vitro and in vivo (Li et al., 2019). HJT-sRNA-m7 was selected from anti-fibrotic herb HJT and targets three fibrotic genes simultaneously: alpha-SMA, fibronectin, and Collagen type III alpha 1 (Li et al., 2019). The design from Mother Nature is fantastic: the bencaosome with HJT-sRNA-m7 improved lung fibrosis both in vitro and in vivo (Li et al., 2019). Furthermore, RNAs resembling microRNAs were identified in Ophiocordyceps sinensis, a renowned traditional Chinese medicine used to stimulate the immune system, decrease blood pressure and inhibit tumor growth (Zhang et al., 2018). The target predictions of these microRNA-like RNAs and GO analysis have confirmed their functions (Zhang et al., 2018). The large amounts of small RNAs we have consumed from plants over time may precisely regulate human gene expression, suggesting that the phrase "You are what you eat" may have scientific foundation. Further studies are necessary to discover sRNAs from herbs that target all human genes, so that a healthy balance of gene expression can be achieved. It appears that Mother Nature may have provided precise remedies for many human diseases.

Useful nucleic acid therapeutics, including antisense oligonucleotides, aptamers, and small interfering RNAs, are understood to inhibit specific functions of particular genes involved in disease. While hundreds of clinical trials are underway around the world, few are approved by the FDA (Vitravene 1998, Macugen 2004, Knamro 2013, Exondys 51 2016, Spinraza 2016, Defitelio 2016). Although quite a few compounds have been shown to provide effective treatment, delivery remains an important hurdle since oligonucleotides do not enter cells by diffusion. In this issue, the bencaosome has been used as a novel therapeutic to deliver oligonucleotides to mice by oral administration, and showed marked treatment effects (Li et al., 2019). By mimicking a decoctosome, the bencaosome could consist of synthetic lipids, sRNAs, chemicals, and proteins targeting different genes in combination to achieve an optimal therapeutic effect. If the problem of oligonucleotide delivery is resolved, the paradigm of the drug market will be shifted: Mother Nature may also provide the drug delivery tools for precision medicine.

Globally, many medicines including Chinese, Ayurveda, Unani, Siddha, Persian, Islamic, Muti, and Mayan, as well as traditional European medicine, were based on herbal treatment with functional components extracted from herbs as the therapeutic agents. Sources of modern pharmaceuticals include chemicals, proteins, and nucleic acids. The variety and richness of sRNAs may provide far more treatment options than chemicals and proteins. Different medicines-be they western or eastern, traditional or modern-may share common modes of action to relieve symptoms and cure disease. Future studies may elucidate the molecular mechanisms of these treatments and allow traditional and alternative therapies to be seen together as one medicine, regardless of origin.

Compliance and ethics The author(s) declare that they have no conflict of interest.

\section{References}

Chin, A.R., Fong, M.Y., Somlo, G., Wu, J., Swiderski, P., Wu, X., and Wang, S.E. (2016). Cross-kingdom inhibition of breast cancer growth by plant miR159. Cell Res 26, 217-228.

Dickinson, B., Zhang, Y., Petrick, J.S., Heck, G., Ivashuta, S., and Marshall, W.S. (2013). Lack of detectable oral bioavailability of plant microRNAs after feeding in mice. Nat Biotechnol 31, 965-967.

Du, J., Liang, Z., Xu, J., Zhao, Y., Li, X., Zhang, Y., Zhao, D., Chen, R., Liu, Y., Joshi, T., et al. (2019). Plant-derived phosphocholine facilitates cellular uptake of anti-pulmonary fibrotic HJT-sRNA-m7. Sci China Life Sci 62, 309-320.

Huang, F., Du, J., Liang, Z., Xu, Z., Xu, J., Zhao, Y., Lin, Y., Mei, S., He, Q., Zhu, J., et al. (2019). Large-scale analysis of small RNAs derived 
from traditional Chinese herbs in human tissues. Sci China Life Sci 62, 321-332.

Khvorova, A., and Watts, J.K. (2017). The chemical evolution of oligonucleotide therapies of clinical utility. Nat Biotechnol 35, 238248.

Li, X., Liang, Z., Du, J., Wang, Z., Mei, S., Li, Z., Zhao, Y., Zhao, D., Ma, Y., Ye, J., et al. (2019). Herbal decoctosome is a novel form of medicine. Sci China Life Sci 62, 333-348.

Majlessi, M., Nelson, N.C., and Becker, M.M. (1998). Advantages of 2'-Omethyl oligoribonucleotide probes for detecting RNA targets. Nucleic Acids Res 26, 2224-2229.

Xin, T., Zhang, Y., Pu, X., Gao, R., Xu, Z., and Song, J. (2018). Trends in herbgenomics. Sci China Life Sci 62, 288-308.
Yu, B., Yang, Z., Li, J., Minakhina, S., Yang, M., Padgett, R.W., Steward, R., and Chen, X. (2005). Methylation as a crucial step in plant microRNA biogenesis. Science 307, 932-935.

Zhang, L., Hou, D., Chen, X., Li, D., Zhu, L., Zhang, Y., Li, J., Bian, Z., Liang, X., Cai, X., et al. (2012). Exogenous plant MIR168a specifically targets mammalian LDLRAP1: evidence of cross-kingdom regulation by microRNA. Cell Res 22, 107-126.

Zhang, W., Li, X., Ma, L., Urrehman, U., Bao, X., Zhang, Y., Zhang, C.Y., Hou, D., and Zhou, Z. (2018). Identification of microRNA-like RNAs in Ophiocordyceps sinensis. Sci China Life Sci 62, 349-356.

Zhu, K., Liu, M., Fu, Z., Zhou, Z., Kong, Y., Liang, H., Lin, Z., Luo, J., Zheng, H., Wan, P., et al. (2017). Plant microRNAs in larval food regulate honeybee caste development. PLoS Genet 13, e1006946.

\section{Biographical sketch}

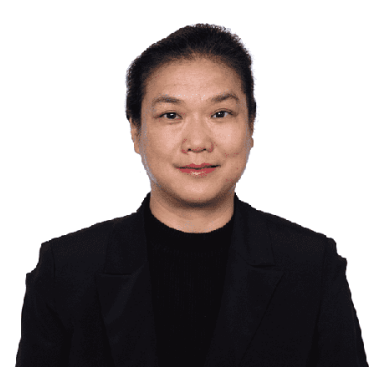

Chengyu Jiang is a professor and Executive Vice Dean of School of Basic Medicine at Peking Union Medical College and Chinese Academy of Medical Sciences. She obtained her B.S. from University of Science and Technology of China and Ph.D. from Brown University followed by postdoctoral training at Massachusetts General Hospital, affiliated to Harvard Medical School before joining PUMC/CAMS in 2003. Her research is to elucidate molecular pathogenesis of Acute Respiratory Distress Syndrome induced by RNA viruses such as SARS-CoV, Avian and Swine Influenza, and Ebola. Her group has also studied molecular mechanism of lung fibrosis, psychiatry and cancer. She has proposed a number of repurposing drugs after elucidation of disease molecular targets. Her recent studies are focused on herbal small RNAs as functional components of medicine. She published extensively in peer-reviewed journals including Nature, Nature Medicine, Molecular Psychiatry, Cell Research, and Nature Communications. She is also an inventor of a number of international patents. Some of the patents are exclusively licensed to top 5 international pharmaceutical companies. She received numerous honors including "Cheung Kong" Scholar, the Young Woman Scientist of China, and National Outstanding Young Award fund. 